\title{
Public Health Observatories: a learning community model to foster knowledge transfer for sustainable cities
}

\author{
Domenico Vito', Manuel Ottaviano², Maria Fernanda Cabrera², José Gabriel Teriús \\ Padrón $^{2}$, Vittorio Casella ${ }^{1}$, Riccardo Bellazzi ${ }^{3}$ \\ ${ }^{1}$ Department of Civil Engineering and Architecture, University of Pavia, Italy. ${ }^{2}$ E.T.S.I. de \\ Telecomunicación, Universitat Politècnica de Madrid, Spain. ${ }^{3}$ Department of Industrial and \\ Information Engineering, University of Pavia, Italy
}

\begin{abstract}
A Public Health Observatory (PHO) is a platform to provide "health intelligence" as a service for a specific population. The World Health Organization (WHO) identifies the primary purposes of PHOs as "monitoring health situations and trends, including assessing progress toward agreed-upon health-related targets; producing and sharing evidence; and, supporting the use of such evidence for policy and decision making" For the purposes of the PULSE project, create an observatory to function as a unique point of access to the PULSE technology for people both inside and outside the project consortium.Specifically, we create a platform for elearning and knowledge sharing that it can be easily navigated by lay persons that are interested in learning about or participating in the PULSE project. We targeted specifically policymakers, clinicians, as well as leaders and citizens in other cities. As a concept, it reflects the principles participation, sustainability, and collaboration across sectors and levels of government The Observatory leverages on the Health in All Policies (HiAP) framework. HiAP is a cross-sectoral approach to public policy that systematically takes into account the health implications of decisions, seeks synergies, and avoids harmful health impacts in order to improve population health and health equity.
\end{abstract}

Keywords: Knowledge Sharing; Public Health; Sustainable Cities 


\section{Introduction}

A Public Health Observatory can be thought of as a platform for providing "health intelligence" for a specific population. The World Health Organization identifies the primary purposes of the Observatories as "monitoring health situations and trends, including assessing progress toward agreed-upon health-related targets; producing and sharing evidence; and, supporting the use of such evidence for policy and decision making"(Leppo et al., 2015).

For the purposes of the PULSE project an Observatory to function as a unique point of access to the PULSE technology for people inside and outside of the project consortium has been created.

The platform that is a user friendly, simple, and straightforward tool so that it can be easily navigated by lay persons that are interested in learning about and/or participating in the PULSE project.

PULSE is a participative project focused on well-being in communities. The final goal is to build extensible models and technologies to predict, mitigate and manage public health problems, and promote population health, in cities-

The project developed a set of tools to disseminate examples of Health in all policies in the seven-participant cities.

PULSE leverages on diverse data sources and big data analytics to transform public health from a reactive to a predictive system, and from a system focused on surveillance to an inclusive and collaborative system supporting health equity.

The types of data are: demographic data across cities, air quality data, health and wellbeing data from questionnaires, data from the PULSE questionnaire/google document survey,air Quality Index (AQI) data from pilot cities,data from PulsAir app and Fitbit, open data; simulation modelling data,dashboards showing real time data, simulation and visualization tools. The public space of the observatory has been designed with the intent of collecting the interests of other stakeholders and cities around the world.

The Observatory manages information gathered through the PULSE technology (risk models and data from air quality sensors and mobile app) and from other external sources available in the test beds

\subsection{The HiAP framework}

Basically, the Observatory is a tool to foster the Health in All Policies (HiAP) framework. HiAP is a cross-sectoral approach to public policy that systematically takes into account the health implications of decisions, seeks synergies, and avoids harmful health impacts in 
order to improve population health and health equity. As a concept, it reflects the principles of legitimacy, accountability, transparency and access to information, participation, sustainability, and collaboration across sectors and levels of government. As such, a broader goal is for the Observatory to be used to help decision makers consider health when implementing policy of any kind.

As defined by the World Health Organization, Health in All Policies (HiAP) is an approach to public policy across sectors that systematically takes into account the health and health systems implications of decisions, seeks synergies, and avoids harmful health impacts, in order to improve population health and health equity. A HiAP approach is founded on health-related rights and obligations. It emphasizes the consequences of public policies on health determinants and aims to improve the accountability of policy-makers for health impacts at all levels of policy-making (Solar et al. 2019).

Solar et al (2009) have proposed a conceptual framework for HiAP implementation, which includes several dimensions and elements involved in the implementation and sustainability of this approach . Firstly, they proposed an explanatory conceptual model of the initiation and implementation of "Health in All Policies" (Figure 1), based on a synthesis of existing literature describing intersectorial and whole-of-government approaches to policymaking in general, and health equity specifically.

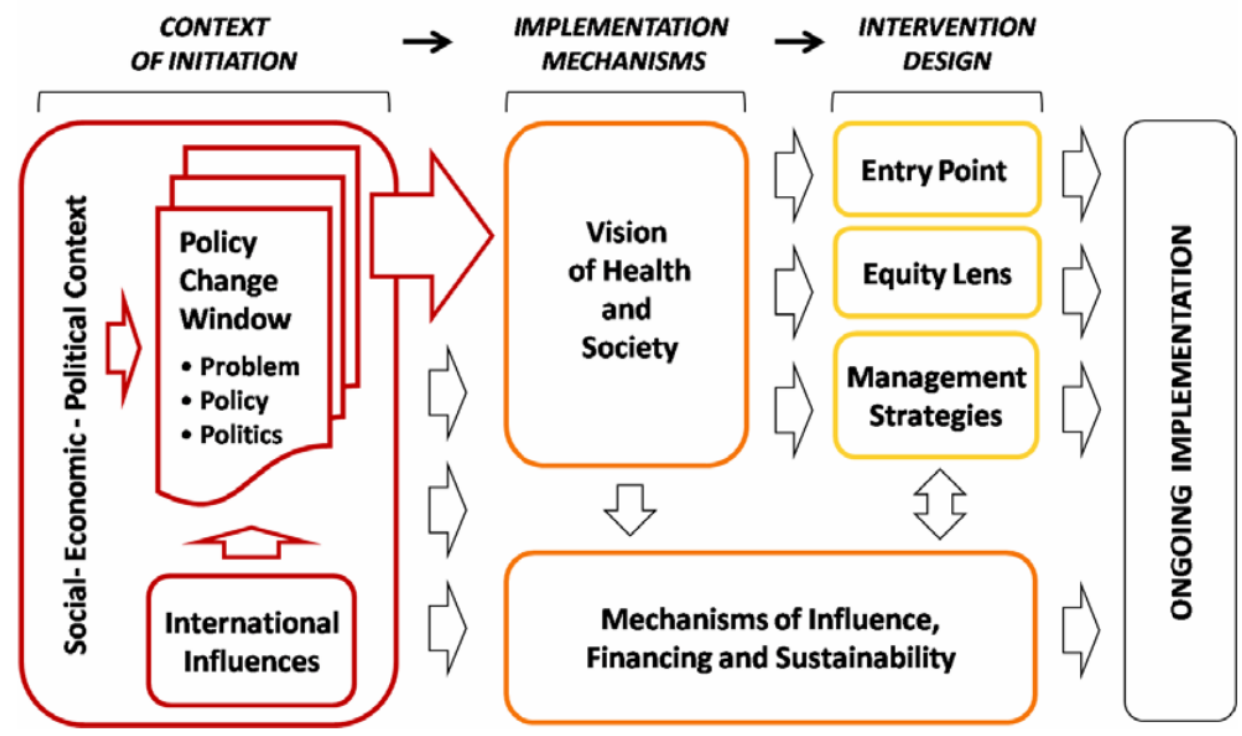

Figure 1.Getting Started with "Health in All Policies": A Conceptual Framework (Solar et al. 2009). 
The framework includes three core conceptual domains, leading to ongoing interventions and policy-making that are mapped into three phases: context of initiation, implementation mechanism and intervention design.

\section{The PULSE Public Health Observatory}

The mission of the PULSE Observatory can be summarized as follows:

- Generating usable knowledge: our goal will be to use the observatory to help to generate knowledge relevant to issues faced by various stakeholders who design, decide, implement or benefit from interventions or policies that aim to improve health

- Facilitating the use of this knowledge: our goal is for stakeholders to use the data from the PULSE study (WebGIS, PulsAir mobile app, Fitbit, and air quality sensors) for the development, monitoring, and evaluation of policies on public health

- Encouraging communication and action based on this knowledge: there is the potential for tremendous benefits from our PULSE Observatory through encouraging dialogue between researchers, public health workers, citizens, and policy-makers about improving the health system.

According to WHO "the link between research and policy is not obvious and the 'transfer' of evidence in public policy requires a good understanding of the political process, taking into account the context in which action takes place." (WHO 2015).

Thus, PHOs plays an active role in brokering knowledge to bridge the gap between knowledge and action between researchers, public health workers, citizens, and policymakers. The study on the structure of PHO in particular addressed the opportunities of the PULSE technology and how the project approach and techniques could be an ideal resource to at least provide a technological substrate to foster HiAP.

\subsection{The Structure of $\mathrm{PHO}$}

The Consortium of PULSE worked to define a structure of the PULSE Public Health Observatory. Figure 2 depicts the most up to date version of the proposed structure for the Observatory.

The work has been carried out with the purpose of creating one unique web-based tool that is able to make visible and integrate all the activities of PULSE (Community of Practice, Learning Platforms, Health in All Policies, etc.), make visible and potentially exploitable the technological components of the project and finally be an official results page for the project.

\subsection{Target groups}

Five types of stakeholders to engage and also the possible technological tools and content available in the project to be shared has been identified for PHO in the following ways. 


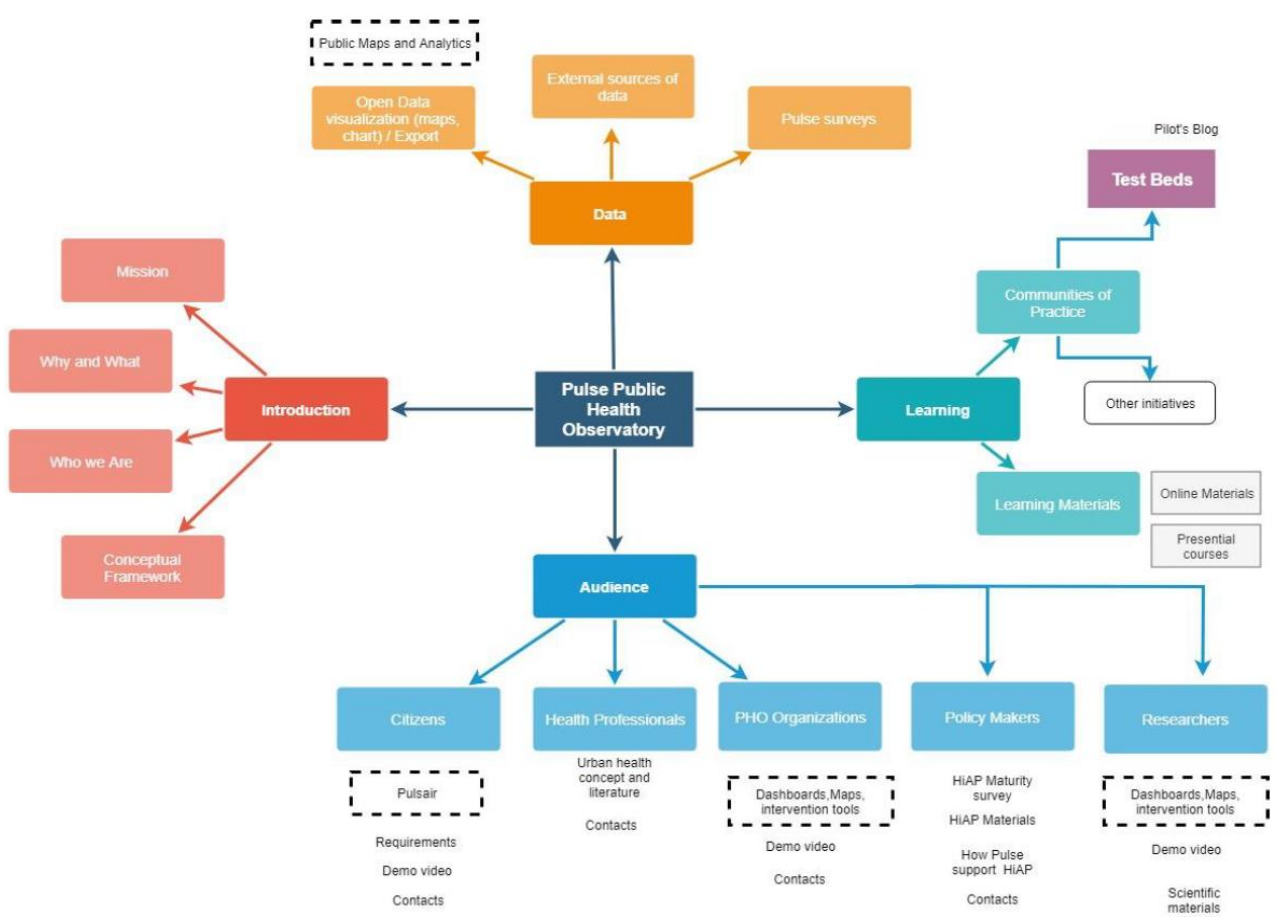

Figure 2. Structure of the PULSE Public Health Observatory.

The citizens are engaged through the mobile app, The Observatory provides general information about the app and its functionalities. All the citizens involved in the project use PulsAir, through which they actively take part in the system and they become both the starting point and the finish line of the data flow. PulsAIR is a novel participatory citizen science-based mobile application used to empower the citizens in their perceiving of urban environmental and health status, to foster a healthy lifestyle and to make people more aware about the air pollution in the city. Participants that consistently use the app and follow its advice are rewarded through a scoring mechanism that also works as an incentive to maintain interest. Through the app, Observatory is able to communicate directly with the population of the city. The web page also contains information about smartphone requirements (minimum operating system and hardware specs of the mobile phone), a demo video, and a contact form to gauge participant usage of PulsAir.

Health professionals are able to access project information and concept as well as literature about urban health.

The Public Health Organizations have access to the PULSE Dashboards and their functionalities (analytics, maps, risk models, simulation. A demonstration video will show 
the full functionality of the tools. Members of the consortium have access to the actual developed Dashboards specific to the city in which they belong.

Policy makers engage with Health in All Policies through published material about the concept and through recommendations from the WHO. There is also be a Maturity Model Scale to assess uptake of an HiAP approach in cities

Furthermore, through the community of Practice, each test bed can share their experiences on how HiAP policies are applied in the city and how PULSE could technically foster the HiAP processes. As with public health organizations, researchers may be interested in dashboard-type tools that can be used to explore epidemiologic data and find correlations with health risks and pollution data.

Access to the Dashboards will allows for the comparison of data between cities (this feature is not accessible by PHO organizations). Furthermore, it will be possible to access the scientific material produced in the context of PULSE and from this initiative.

\section{Learning Tools of PHO}

The users of the Observatory can learn about the implementation strategies used by each pilot during the project. This includes a menu for each pilot city on the website where users can get information about the city as well findings from the study.

Although the final aim of PULSE is to create a versatile and adaptive platform that can potentially be applied to any urban environment, seven big cities across the globe were selected to test the PULSE system. These cities are Barcelona, Birmingham, New York City, Paris, Singapore, Pavia and Keelung. In each city, the app and the Fitbits are deployed to gather health and mobility data, in a unified format, from the population. However, each city has a differing access to public health and open data. Available data is integrated in the WebGIS and are being used to create specific dashboards and risk models for each pilot site. Moreover, air quality is measured differently in each city, through an integration of pre-existing monitoring stations and new air quality sensors purchased ad hoc during the project.

The Public Health Observatory proposes two approaches of learning:

- $\quad$ The Community of Practice (CoP) which can be used by each test bed (seven in total) to share information about deployment, focus of the pilot, and lessons learned. This section also contains experiences from other cities that are not a part of the project.

- Learning Materials: the Public Health Observatory also hosts a course on Big Data to Policy (BD2P) to train users to inform and shape urban policy across the health sector. Environment and transport content will be further developed in task 8.4 (PULSE Digital Training and Education Modules: Big Data to Policy). This material 
will be digital, and it will also be possible to assist specific seminars (this is still under discussion among the PULSE partners).

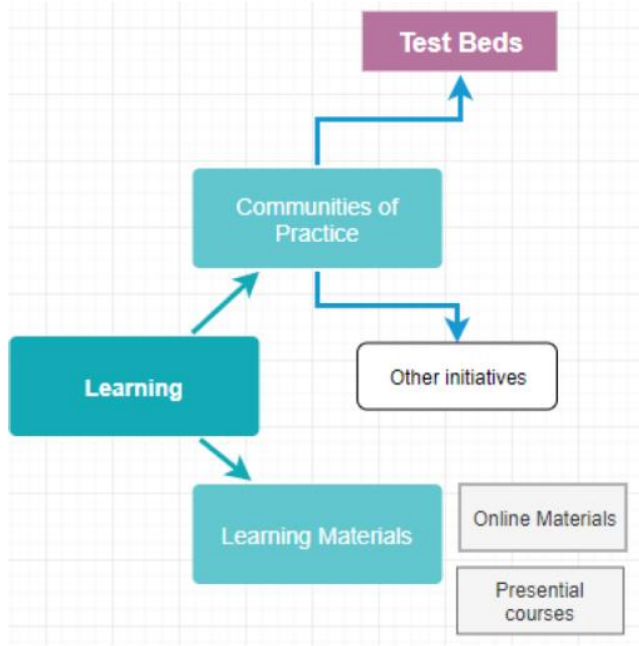

Figure 4. Schema of the learning services provided by the Public Health Observatory

\subsection{Community of practice}

The community of practice is intended as a web page shares all the information and news related to the test bed activities.

The contents are information about recruitment in each city, publications and conferences, content related to PULSE, video testimonials, interviews, preliminary pilot results (dynamic number of enrolled citizens, Air Quality (AQ) average in the cities, etc.), relevant content and initiatives from other online resources, links to training materials that are available on the WHO website.

\subsection{Learning material: the new course Big Data to Policy (BD2P) course}

The new course Big Data to Policy (BD2P) was defined and developed during WP8 of the PULSE project and has been subjected to an external review by market consultants (education) retained by Ohio University. These consultants have provided interim feedback indicating that the BD2P course is unique within offerings on the open market, especially given that the course is tailored to students who are not trained in data science.

It has a specific relevance to policy makers, and city officials, whose portfolios address a wide range of issues, including but not limited to sustainability, the climate neutral city, new mobility services and models, and population health and wellbeink. It fills a niche in urban design and planning and will be of interest to current students in further education, as well as practitioners in the field. 
The course is subject to rigorous review and evaluation of user experience and satisfaction, and that the course be extended, over time, into adjacent areas of interest to potential students. The metrics to assess the BD2P course includes perceived usefulness, clarity, easiness of the educational material, feedback on the workload of the course, missing or expected contents, suggestions on how to improve the course.

\section{Conclusions}

The work reported the activities related to the definition, specification of the PULSE Public Health Observatory. These tools have been conceived as a comprehensive instrument available for different types of audiences (citizens, health care providers, PHOs, researchers, and policy makers) to learn and use data to take decision for public health. The paper describes the public accessible structure of the observatory that represent the framework of the project, how the project can foster the HiAP frameworks with the digital tools (Dashboards, CoP). The PULSE project also considers relevant to work on a final validation plan and present all the studies that will be performed in the remaining months.

\section{Acknowledgement}

PULSE project has been founded by the European Union's Horizon 2020 research and innovation programme, and it is documented in the grant agreement No 727816 . Specifically. PULSE has been founded under the call H2020-EU-3.1.5. in the topic SCIPM-18-2016-Big Data supporting Public Health policies. More information on http://www.project-pulse.eu

\section{References}

Leppo K., Ollila E, Peña S, Wismar M, Cook S. Health in All Policies: Seizing opportunities, implementing policies. Helsinki: Ministry of Social Affairs and Health, Finland; 2013. 350 p.

Solar, O., Valentine N., Rice M., \& Albrecht D. (2009). Moving forward to equity in health: what kind of intersectoral action is needed? An approach to an intersectoral typology. 7th Global Conference on Health Promotion, "Closing the Implementation Gap. ” Nairobi, Kenya

WHO (2015) Intersectoral Action and Health Equity in Latin America: An Analytical Approach Available from: http://iris.paho.org/xmlui/bitstream/handle/ 123456789/33873/9789275118573-eng.pdf?sequence=1\&isAllowed=y 